\title{
Microlensing pulsars
}

\author{
Shi Dai and Renxin Xu \\ Department of Astronomy, School of Physics, Peking University, Beijing 100871, China \\ email: daishi@pku.edu.cn, r.x.xu@pku.edu.cn
}

\begin{abstract}
We propose to determine the mass of isolated neutron stars through gravitational microlensing. We show that the all-sky microlensing pulsar event rate is $\sim 2.8 \times 10^{-10}$ per year per background source (/yr/source). Microlensing neutron star event rate would contribute $\sim 20 \%$ to the total Galactic event rate at time-scale of $\sim 15$ days. We also present catalogue comparisons between known pulsars and background stars. We find that several pulsars would pass by background stars closely and may cause observable astrometric microlensing phenomenon. According to our covariance analysis, the uncertainty of masses determined through astrometric microlensing could be $\sim 20 \%$. Therefore, gravitational microlensing is a promising way to determine the mass of isolated neutron stars with future advanced radio and optical telescopes.
\end{abstract}

Keywords. gravitational lensing, pulsars: general, stars: neutron

\section{Introduction}

The measurement of neutron star masses is essential to understand the inner structure of neutron stars and the state of dense matter at nuclear density. However, up to now, all accurate mass measurements are from observations of binary pulsars. As for isolated neutron stars, the mass measurement is still challenging. Gravitational microlensing has been suggested to measure the mass of isolated neutron stars (e.g., Dai et al. 2010). Future advanced radio and optical telescopes will greatly enhance the possibility of discovering microlensing pulsar events.

In Dai \& Xu (2013, in prep.) we show that the all-sky microlensing pulsar event rate is $\sim 2.8 \times 10^{-10} / \mathrm{yr} /$ source, and it could be 100 times larger for astrometric microlensing. Considering the kick velocity, the fractional contribution of microlensing neutron star event rate to the total Galactic event rate could be up to $\sim 20 \%$ at time-scale of $\sim 15$ days. Therefore, it is hopeful to discover microlensing pulsar events. We also present catalogue comparisons between known pulsars, microlensing candidates and background stars. We find that several known pulsars would pass by background stars closely and may cause observable astrometric microlensing phenomenon. The covariance analysis of the simulated observations shows that the masses of neutron stars can be determined accurate to $\sim 20 \%$.

\section{Event rates and time-scale distributions}

We calculated the microlensing pulsar event rates, based on the spatial and velocity distribution of pulsars (Faucher-Giguère \& Kaspi 2006). For bulge, the all-sky event rate is $\sim 1.1 \times 10^{-10} /$ year/source, and for disk, it is $\sim 1.7 \times 10^{-10} /$ year/source. The total event rate is $\sim 2.8 \times 10^{-10}$ /year/source, and it could be $\sim 100$ times larger for astrometric microlensing since the cross-section of astrometric microlensing is much larger than that of photometric microlensing. On the other hand, considering the kick velocity of neutron stars, we show that the fractional contribution of microlensing neutron star event rate to the total Galactic event rate could be up to $\sim 20 \%$ at time-scale of $\sim 15$ days (see 


\begin{tabular}{|c|c|c|c|c|}
\hline Pulsar & \begin{tabular}{|c|} 
Background source \\
ID(UKIDSS)
\end{tabular} & \begin{tabular}{|c|} 
Current \\
centroid shift \\
$(\mu \mathrm{as})$
\end{tabular} & $\begin{array}{c}\text { Maximum } \\
\text { centroid shift } \\
(\mu \mathrm{as})\end{array}$ & $\begin{array}{l}t_{0} \\
(\mathrm{yr})\end{array}$ \\
\hline $\mathrm{J} 1910+1256$ & 43868 & 1 & $27 t$ & $551(9)$ \\
\hline B1929+10 & 438809433778 & 8.9 & $216(35)$ & $35(0.03)$ \\
\hline J1835-1106 & 438519400030 & 1.3 & $173(151512)$ & $-47(130)$ \\
\hline B1829-08 & 438747104229 & 0.58 & $146(8869)$ & $-204(148)$ \\
\hline B1952+29 & 438604464609 & 15 & $124(233)$ & $42(13)$ \\
\hline
\end{tabular}

Table 1. Pulsars and background sources with maximum centroid shift larger than 100 mas.

Fig 1). Surveys such as LSST will discover huge amounts of microlensing events, then it is possible to search pulsars among those short duration events and determine their masses at the same time.

\section{Catalogue comparison}

First, we compared the positions of pulsars and that of microlensing candidates. We used the ATNF pulsar catalogue (Manchester et al. 2005), which contains 2008 pulsars. The positions of microlensing candidates are from the published results of EROS (Hamadache et al. 2006), MACHO (Thomas et al. 2005), MOA $\dagger$ and OGLE (Udalski 2003). We found no known pulsars associated with identified microlensing candidates. Next, we carried out catalogue comparisons between known pulsars, SDSS and UKIDSS sources. We found that several pulsars would pass by background stars closely and may cause observable astrometric microlensing phenomenon. In Table 1, we list relevant information of pulsars and their associated background sources with maximum centroid shift larger than 100 uas.

We used covariance analysis to estimate the uncertainty of masses determined through astrometric microlensing phenomenon. In Fig 2, we show the percentage error of Einstein radius as a function of number of data points for a typical microlensing pulsar event.

$\dagger$ http://www.phys. canterbury.ac.nz/moa/index.html

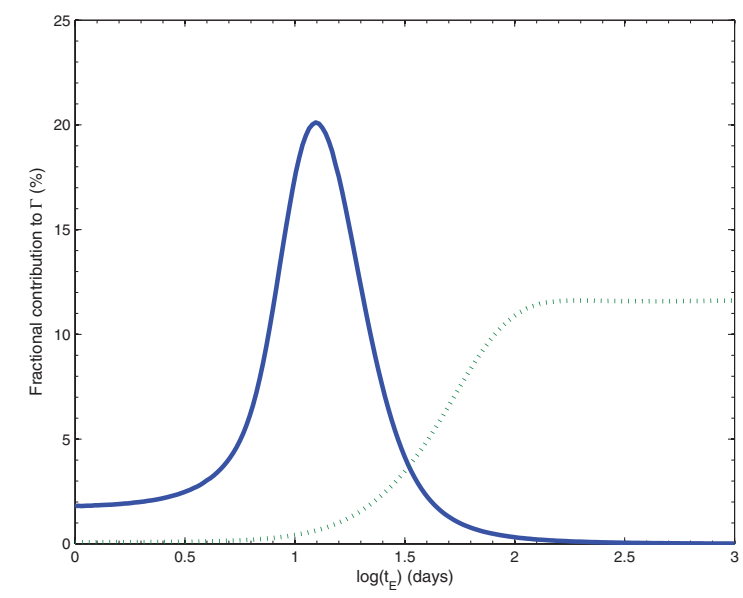

Figure 1. Fractional contribution of microlensing neutron stars event rate to the total Galactic event rate, as a function of the event time-scale. The solid and dashed lines represent calculations considering the kick velocity of neutron stars and without considering, respectively. 


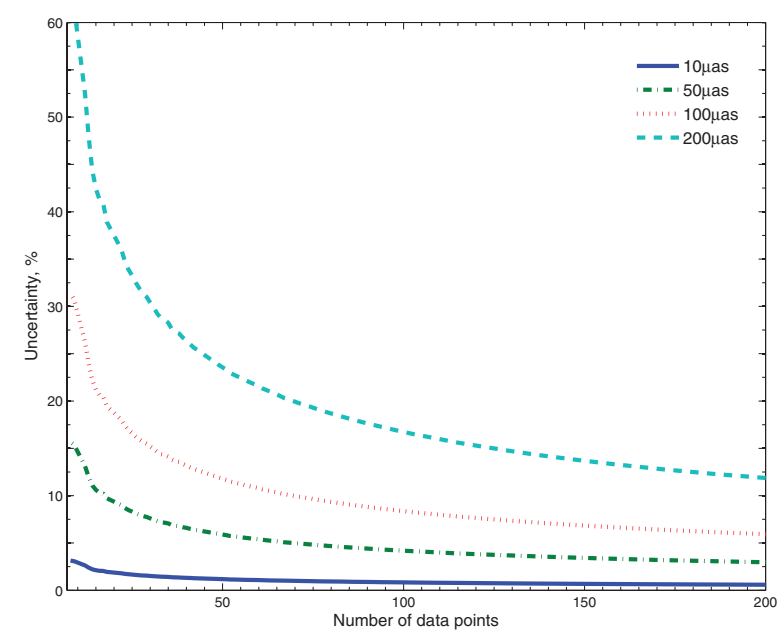

Figure 2. The percentage error of Einstein radius as a function of number of data points.

Different lines represent different astrometric accuracy.

With the definition of Einstein radius, $\theta_{E}=\sqrt{\frac{4 G M\left(D_{\mathrm{s}}-D_{\mathrm{d}}\right)}{c^{2} D_{\mathrm{s}} D_{\mathrm{d}}}}$, and taking the uncertainty of Einstein radius and distance to pulsars to be $5 \%$ and $10 \%$, respectively, we can determined the masses of pulsars accurate to $\sim 20 \%$.

\section{Conclusion}

Gravitational microlensing could be a promising way to determine the mass of isolated neutron stars. The all-sky microlensing pulsar event rate is $\sim 2.8 \times 10^{-10}$ /year/source, and it could be much larger for astrometric microlensing. Considering the kick velocity, microlensing neutron star events could contribute up to $\sim 20 \%$ of Galactic events at timescale of $\sim 15$ days. Catalogue comparisons between known pulsars and background stars showed that several pulsars may cause observable astrometric microlensing phenomenon, and the masses can be determined accurate to $\sim 20 \%$.

\section{Acknowledgements}

This work is supported by the National Basic Research Program of China (2012CB821800, 2009CB824800), the National Natural Science Foundation of China (Grant Nos. 10935001, 10973002) and the John Templeton Foundation.

\section{References}

Belokurov, V. A. \& Evans, N. W. 2002, MNRAS, 331, 649

Dai, S., Xu, R. X., \& Esamdin, A. 2010, MNRAS, 405, 2754

Hamadache C., et al. 2006, A\&A A, 454, 185

Faucher-Giguère, C. \& Kaspi, V. M. 2006, ApJ, 643, 332

Manchester, R. N., Hobbs, G. B., Teoh, A., \& Hobbs, M. 2005, AJ, 129, 1993

Thomas, C. L. et al. 2005, ApJ, 631, 906

Udalski, A. 2003, AcA, 53, 291 